TITLE:

\title{
Natural disasters and dynamics of "a paradise built in hell": a social network approach
}

AUTHOR(S):

Kotani, Hitomu; Yokomatsu, Muneta

\section{CITATION:}

Kotani, Hitomu .... [et al]. Natural disasters and dynamics of "a paradise built in hell": a social network approach. Natural Hazards 2016, 84(1): 309-333

ISSUE DATE:

2016-10

URL:

http://hdl.handle.net/2433/226619

\section{RIGHT:}

The final publication is available at Springer via https://doi.org/10.1007/s11069-016-2432-8; The full-text file will be made open to the public on 1 October 2017 in accordance with publisher's 'Terms and Conditions for Self-Archiving'.; この論文は出版社版でありません。引用の際には出版社版をご確認ご利用ください。; This is not the published version. Please cite only the published version. 


\title{
Natural Disasters and Dynamics of "A Paradise Built in Hell": A Social Network Approach
}

\author{
Hitomu Kotani · Muneta Yokomatsu
}

Received: 16 February 2016 / Accepted: 15 June 2016

\begin{abstract}
Solnit (2010) notes that it is often the case that immediately after a disaster occurs, voluntary mutual help motivated by altruism among the victims is observed. She names this phenomenon "a paradise built in hell." Subsequently, it has been pointed out that "a paradise built in hell" has the potential to reform existing social institutions in the long term. Through the application of a social network model based on game theory, this study models the link formation motivated by altruistic preferences during disasters and analyzes the possibilities for a long-term outcome induced by the short-term effect of "a paradise built in hell." More specifically, we utilize numerical simulations and examine the dynamic effect of altruistic link formation during disasters on the properties of a network such as network density and disparities in the number of links of each player. In addition, this study focuses on larger-scale disasters that lead to more instances of altruistic behaviors among affected people, and analyzes such behaviors' cross-sectional and dynamic effects on social welfare as well as the possibility of the long-term outcome of "a paradise built in hell."
\end{abstract}

Keywords Social networks - A paradise built in hell - Network formation model · Altruism · Dynamic effect

\section{Introduction}

Extraordinary events often result in people having unusual opportunities to interact with others. This is because such events place people into contexts or roles that are different from those of regular life, such as at work or school. Kotani and

\author{
Hitomu Kotani \\ Graduate School of Engineering, Kyoto University, Gokasho, Uji, Kyoto, Japan \\ Tel.: +81-774-38-4038 \\ Fax: +81-774-31-8294 \\ E-mail: hitomukotani@drs.dpri.kyoto-u.ac.jp \\ Muneta Yokomatsu \\ Disaster Prevention Research Institute, Kyoto University, Gokasho, Uji, Kyoto, Japan
}


Yokomatsu (2015a) showed that the random rewiring of links caused by extraordinary events has a long-term effect on the growth of social networks, and can result in an increase in individual utility.

Disasters can be viewed as extraordinary events (e.g., Fujimura 2001). Disasters force people to relinquish their usual behavioral patterns, and instead follow different patterns. For example, one could assume that people prefer to interact with others who have many friends in regular life, because through the resulting links people are more likely to expand their opportunities for finding jobs or other personal gains. This preference would allow people to expand their networks. However, if a disaster occurs, people are concerned about and contact those who have fewer friends, because they might believe that those who have many friends will be supported by them.

Solnit (2010) notes that it is often the case that immediately after a disaster occurs anywhere in the world, voluntary mutual help among the victims is observed, motivated by altruism. She names this phenomenon as "a paradise built in hell," which is also called by other researchers like "post-disaster utopia," "altruistic or therapeutic community," and "democracy of distress" by disaster researchers (e.g., Wolenstein 1957; Barton 1969; Giel 1990; Kaniasty and Norris 2004). For example, Solnit (2010) gives an example of a Hurricane Katrina victim evacuated to an elementary school shelter, who said, "I saw people being compassionate about people that they never met, people that they never saw, people that they never knew reaching out them, feeding them, giving them clothes. ... This was everywhere in New Orleans." Moreover, Kotani and Yokomatsu (2015b) conducted an interview survey in the area affected by the Great Hanshin-Awaji Earthquake that struck Japan in 1995 and revealed that during a water failure after the quake, well water at a food market was supplied not only to local residents who had interacted previously in the market but also to those who had not.

Subsequently, some works (e.g., Ohsawa 2011; Yamori 2011) have pointed out that "a paradise built in hell" has the potential to reorganize the existing society, and to result in the creation of an alternative society in the long term. Hence, "a paradise built in hell" would represent both a short-term aspect, the mutual help motivated by altruism, and a long-term one, the reform of the society. However, the general process and condition under which the short-term aspect eventually becomes long-term remain unclear. To answer the questions, this study analyzes the possibilities for the long-term development induced by the short-run effect of "a paradise built in hell," through the application of a social network model. As previously mentioned, distinct behavioral principles have been observed depending on whether people are in a time of peace or experiencing a disaster, and thus, the model assumes that a player has a selfish, or non-altruistic, preference in peaceful times and an altruistic one during disasters. Then, we conduct numerical simulations of the model and examine the dynamic effect of altruistic link formation during disasters on the properties of a network, such as network density and disparity in the number of links of each player.

The rest of this paper is organized as follows. Section 2 presents the literature review and outlines the contribution of this paper. Section 3 formulates the network formation model. Section 4 analyzes network formation in cases with and without the presence of disasters by means of numerical simulations, focusing on the different cost functions for communication. Section 5 extends the model so as to consider many players are affected by disasters, and analyzes its network 
formation. Section 6 discusses the results obtained in Sections 4 and 5. Section 7 concludes our findings and describes future work.

\section{Literature review}

Research on social network formation after a disaster is classified roughly into two types: 1) qualitative analysis based on methods in sociology, psychology, or ethnography, and 2) mathematical analysis based on the graph theory. The former has mainly concentrated on a detailed description of phenomena that occur immediately after disasters, on the basis of information gained from interviews and/or field surveys conducted after the event. For example, previous studies have analyzed deepening kinship ties immediately after a hurricane (e.g., Fogleman and Vernon 1977), the ties that emerge immediately after a hurricane among people in a minority community who did not know or interact with each other before the event took place (e.g., Messias et al. 2012), a pay-it-forward network to survivors of a current disaster created by support from survivors of previous disasters (e.g., Atsumi 2014), and so forth.

The latter, namely, the approach using mathematical network models, has the advantage of dealing with more individuals simultaneously. In general, such mathematical approaches are classified into two categories: 1) statistical and empirical analyses with real data, and 2) qualitative structural analyses of possible future issues by means of analytical or simulation approaches. As an example of the former, Phan and Airoldi (2015) analyzed the effects of Hurricane Katrina on a social network of students both in affected and non-affected areas, leveraging data from a sample of 1.5 million college students who wrote messages and posts on Facebook over four years. They estimated the short-term (four weeks after the Hurricane) and subsequent (fifty-two weeks after the event) effects of Katrina on social network formation, and also estimated changes in the friendship formation pattern of the affected students after Katrina. The results suggest that affected students are less prone to "preferential attachment dynamics" (i.e., the well-known friendship formation process in which friendships are chosen proportional to degree), thus displaying a shift from a typical friendship formation pattern. While Solnit (2010) remarked upon the existence of altruistic behavior immediately after a disaster, our study goes a step further and models the above-mentioned altruistic behavior as a behavior that "lets one connect to persons with less relationships immediately after disasters." Phan and Airoldi (2015) have shown that our proposed model is reasonable.

Although our study follows the mathematical simulation approach, we use only hypothetical parameters, and thus, we are unable to quantitatively predict possible future phenomena. Instead, we can reduce the elements in the model as much as possible and clarify the theoretical consequences of the factors in the model. In addition, compared to statistical analysis, our approach makes it relatively easy to conduct a dynamic analysis on the longer-term aspects. Our simulation approach reveals that altruistic behavior immediately after a disaster has both negative cross-sectional externality and positive dynamic externality.

As mentioned above, our model assumes that an individual's preference changes depending on whether he/she is in a time of peace or experiences a disaster. During the former, persons are assumed to have selfish or non-altruistic, preferences 
with regard to obtaining more information and making new connections by linking with those who already have many links. For the sake of simplicity, we call this preference "selfish preference" in the rest of the paper. On the other hand, during a disaster, persons are assumed to have altruistic preferences for linking with those who have almost no links in order to help them. Among previous social network models, Chasparis and Shamma (2013) alone introduced the state-dependent utility function. According to their model, the amount of the link establishment cost of player $i$ varies according to state $x_{i}$ that $i$ belongs to. In contrast, in our model, the form of the utility function itself changes depending on times of peace and disasters. In addition, we assume that such preference changes are exogenous shocks that are unpredictable and uncontrollable for the players. Then, we investigate the possibility that the behaviors during a disaster temporarily reduce social welfare measured by utilities with selfish preference, but the behaviors increase the social welfare long after a disaster. To the best of our knowledge, our study is the first to employ the above-mentioned modeling with the stated aims.

\section{Network Formation Model with a Focus on Times of Peace and Disasters}

\subsection{The Model}

Let $N=\{1, \cdots, n\}$ be the finite set of players who are community members $(n>$ 2 ) in a community. The network relations among the players are represented by graphs, whose nodes represent the players and whose links capture the connections between the players. A network $g$ is a set of links between the players in $N$. A link connecting players $i$ and $j$ is represented by $i j$. If $i j \in g$, then players $i$ and $j$ are directly connected in the network $g$, while if $i j \notin g$, then the players are not directly connected. For example, if $n=3$ and there are directed links between players 1 and 2 and players 2 and 3, then the network is written as $g=\{12,23\}$. In addition, let $g+i j$ denote the network obtained by adding link $i j$ to the existing network $g$ and let $g-i j$ denote the network obtained by deleting link $i j$ from the existing network $g$, that is, $g+i j=g \cup\{i j\}$ and $g-i j=g \backslash\{i j\}$.

Player $i$ 's neighbors are given by all $j \in N$ such that $i j \in g$. In this model, neighborly relation is defined by a certain level of communication, meetings and sharing of information. Formally, the set of neighbors of $i$ in $g$, denoted by $N_{i}(g)$, is defined as $N_{i}(g)=\{j \in N \mid i j \in g\}$. The degree of player $i$ is the number of neighbors of $i$, which is defined as $d_{i}(g)=\left|N_{i}(g)\right|$.

Given a network $g$, we now define the utility function of player $i$ as follows:

$$
\begin{array}{r}
u_{i}^{h}(g)=\sum_{j \in N_{i}(g)} b^{h}\left(d_{j}\right)-c\left(d_{i}\right) \cdot d_{i} \\
(h=s, a)
\end{array}
$$

where $c\left(d_{i}\right)$ in the second term represents the cost to $i$ of interacting with a neighbor. In the following, we will call $c\left(d_{i}\right)$ the "communication cost." In general, the larger the number of neighbors of $i$, the more the schedule adjustment and the opportunity cost seem to increase for $i$, so $c\left(d_{i}\right)$ is assumed to be a non-decreasing function. That is, $\frac{d c\left(d_{i}\right)}{d d_{i}} \geq 0$. By $h$ we denoted the type of preference of a player. 
Explicitly, $h=s$ denotes that player $i$ has a "selfish preference," and $h=a$ denotes an "altruistic preference." During a time of peace, players are assumed to have a selfish preference $(h=s)$, which leads them to interact with others who have more neighbors in order to expand their opportunities for jobs or other personal gains and fun. Then, $b^{s}\left(d_{j}\right)$ denotes the benefit obtained from neighbor $j$ in a time of peace, such that $\frac{d b^{s}\left(d_{j}\right)}{d d_{j}}>0$. In contrast, during a disaster, and given a pair $i j$ that are affected by the disaster, we identify the player with the larger degree as player $i$. We assume that $i$ now has an altruistic preference $(h=a)$, which leads to $i$ interacting with those who have fewer neighbors. Then, $b^{a}\left(d_{j}\right)$ denotes the benefit obtained from neighbor $j$ during a disaster, such that $\frac{d b^{a}\left(d_{j}\right)}{d d_{j}}<0$. Here, the preference of the player $j$ with the smaller degree is assumed to remain as $h=s$. Appendix A summarizes variables that appear in the model in Sections 3-5.

\subsection{The Dynamic Process}

\subsubsection{The Case without Disaster}

In the community, $n$ players, who are community members, play a game repeatedly. Let $g^{t}$ denote the network at the beginning of period $t(t=1,2, \cdots)$. Given $g^{t}$, players with selfish preferences $h=s$, namely preferences in time of peace, decide to add or delete links.

Our dynamic process of link formation is an example of a dynamic process of network formation accompanied with exogenous randomness, which has been widely used in many papers (e.g., Jackson and Watts 2002a, b). This means that the process assumes bounded rationality of players: the inertia, myopic, and error/mutation hypothesis. In the situation that we consider, these properties are satisfied as follows:

1. Not all players need to react instantaneously to their environment (the inertia hypothesis).

2. Players react myopically when they react (the myopic hypothesis).

3. There is a small probability that players change their strategies at random (the error/mutation hypothesis).

Unintended changes in a network caused by errors/mutations are due to the exogenous factors that are independent of individual rational choice such as loss of common jobs or hobbies, malfunction of communication means and unexpected assignment of roles of community activities where two community members start communication. Without the presence of errors or mutations, one equilibrium is locked in at random by externality. However, under our process the network continues to change indefinitely and to visit each network over time, so that the process identifies the most robust or easy-to-reach networks in the long run.

The dynamic process in period $t$ consists of the following four steps, as proposed by Jackson and Watts (2002a).

1 st

A pair of players $i j$ is randomly identified with fixed probability distribution $\left\{p_{i j}\right\}$ where $p_{i j}>0$ for each $i j$. 
2nd

The remaining players (i.e., all players except the pair $i j$ ) do not react instantaneously to their environment. In other words, do not adjust their links (the inertia hypothesis). 3rd

The pair $i j$ with selfish preferences $(h=s)$, identified in the first step, myopically decide whether to add or delete the link $i j$, following the rule shown in steps 3-1 and 3-2 below, which we call "two sided-link formation" (the myopia hypothesis). They make this choice based on the assumption that all players (including themselves) will have the same links (other than the link $i j$ ) as at the beginning of this period.

3-1 In case that the link $i j$ is not in the network $g^{t}$, it is added if at least one player's utility strictly increases and the other player's does not decrease. Otherwise, it is not added.

3-2 In case that the link $i j$ is already in the network $g^{t}$, it is deleted if either player would strictly benefit from its removal. Otherwise, it remains connected.

4 th

After the choice is made, there is some small probability $\epsilon(0<\epsilon<1)$ that an error/mutation occurs (the error/mutation hypothesis). Hence, the choice made in the third step is not reversed with probability $1-\epsilon$, and is reversed with probability $\epsilon$. This process determines the network $g_{1}^{t}$.

More specifically, the network $g_{1}^{t}$ is obtained as follows.

- In case $i j \notin g^{t}$ If $u_{i}^{s}\left(g^{t}+i j\right) \geq u_{i}^{s}\left(g^{t}\right)$ and $u_{j}^{s}\left(g^{t}+i j\right) \geq u_{j}^{s}\left(g^{t}\right)$ with one inequality strict, then $g_{1}^{t}=g^{t}+i j$ with probability $(1-\epsilon)$ and $g_{1}^{t}=g^{t}$ with probability $\epsilon$. Otherwise, $g_{1}^{t}=g^{t}$ with probability $(1-\epsilon)$ and $g_{1}^{t}=g^{t}+i j$ with probability $\epsilon$.

- In case $i j \in g^{t}$

If $u_{i}^{s}\left(g^{t}-i j\right)>u_{i}^{s}\left(g^{t}\right)$ and/or $u_{j}^{s}\left(g^{t}-i j\right)>u_{j}^{s}\left(g^{t}\right)$, then $g_{1}^{t}=g^{t}-i j$ with probability $(1-\epsilon)$ and $g_{1}^{t}=g^{t}$ with probability $\epsilon$. Otherwise, $g_{1}^{t}=g^{t}$ with probability $(1-\epsilon)$ and $g_{1}^{t}=g^{t}-i j$ with probability $\epsilon$.

The network $g_{1}^{t}$ that is determined through the above process constitutes the network at the end of period $t$. This network is also the network at the beginning of period $t+1$. After period $t+1$, the above process is repeated.

Note that in the third step, one can see the asymmetry of adding and deleting a link. This represents the fact that the formation of a link requires the consent of both players, but severance can be carried out unilaterally.

\subsubsection{The Case Including Disasters}

Let us assume the occurrence of a disaster where the community members $i$ and $j$ who were identified in the first step of the dynamic process that was presented above are affected. We add the following fifth step. 
In every period, disasters occur with probability $\eta(0<\eta<1)$. If no disasters occur, with probability $1-\eta$, then the fifth step ends.

When a disaster occurs, then, redefine the player with the larger degree from the pair $i j$ identified in the first step as player $i$. In other words, redefine players $i$ and $j$ such that $d_{i}>d_{j}$. If $g_{1}^{t}$ of the fourth step does not include the link $i j$, then player $i$ is furnished with an altruistic preference $h=a$ and player $j$ with a selfish preference $h=s$, and they decide whether or not to add the link $i j$ based on the notion of two sided-link shown in the steps 3-1 and 3-2.

Similarly to the fourth step, there is some small probability $\epsilon$ that an error/mutation occurs in the fifth step, which reverses the choice.

Consequently, if the link $i j$ is added, then player $i$ deletes the link $i k$ with the player $k$ that has the largest degree among $i$ 's neighbors. In other words, $k$ is identified such that $\left\{d_{k} \geq d_{l}\right.$ for $\left.\forall l \in N_{i}\left(g_{1}^{t}\right)\right\}$. Through the above process, the network $g_{2}^{t}$ is determined, and also becomes the network at the beginning of period $t+1$, namely, $g^{t+1}$.

More specifically, the network $g_{2}^{t}$ is obtained as follows.

$g_{2}^{t}=g_{1}^{t}$ happens with probability $1-\eta$.

Otherwise, the following process (A) happens with probability $\eta$.

- In case $d_{i} \neq d_{j}$

Redefine players $i$ and $j$ such that their degrees satisfy $d_{i}>d_{j}$. Moreover, define another player $k$ in the set of the neighbors of player $i$ such that $d_{k} \geq d_{l}, \forall l \in N_{i}\left(g_{1}^{t}\right)$. If more than one player satisfies the condition to be player $k$, select one randomly from among them, and define that player as $k$.

- In case $i j \notin g_{1}^{t}$

If $u_{i}^{a}\left(g_{1}^{t}+i j\right) \geq u_{i}^{a}\left(g_{1}^{t}\right)$ and $u_{j}^{s}\left(g_{1}^{t}+i j\right) \geq u_{j}^{s}\left(g_{1}^{t}\right)$ with one inequality strict, then $g_{2}^{t}=g_{1}^{t}+i j-i k$ with probability $(1-\epsilon)$ and $g_{2}^{t}=g_{1}^{t}$ with probability $\epsilon$. Otherwise, $g_{2}^{t}=g_{1}^{t}$ with probability $(1-\epsilon)$ and $g_{2}^{t}=g_{1}^{t}+i j-i k$ with probability $\epsilon$.

- In case $i j \in g_{1}^{t}$ $g_{2}^{t}=g_{1}^{t}$.

- In case $d_{i}=d_{j}$ $g_{2}^{t}=g_{1}^{t}$.

Note that this model deletes the link $i k \in g_{1}^{t}$ if the link $i j$ is added during a disaster. This process arises for the following two reasons. The first relates to the modeled phenomena. When player $i$ obtains an altruistic preference during a disaster and interacts with a player $j$ that has a smaller degree, then $i$ expects the player $k$ that has the largest degree to be supported by some of $k$ 's many friends, so $i$ would stop allocating time for interactions with $k$. The second is to make a comparative analysis clear. Adding one link in the fifth step without deletion derives a natural result that the number of links in the network is increased by that operation. However, our aim is to investigate the true long-term effects of adding a link in the fifth step on network formation, so in order to avoid this obvious result, we remove link $i k$ and exclude the short-term effects that are directly brought by the fifth step operation. 


\section{Numerical Simulation}

\subsection{Functions and Parameters}

Let us specify the functions and parameters as follows:

$$
\begin{aligned}
& b^{s}\left(d_{j}\right)=d_{j}-1, \\
& b^{a}\left(d_{j}\right)=n-d_{j}, \\
& c\left(d_{i}\right)=\bar{c} \cdot d_{i}^{\alpha}, \bar{c}=0.2, \\
& p_{i j}=\frac{1}{\left(\begin{array}{c}
n \\
2
\end{array}\right)}, g^{1}=\emptyset, \\
& n=20, T=3000, \\
& \epsilon=0.05, \eta=0.1 .
\end{aligned}
$$

The communication cost $c\left(d_{i}\right)$ would be affected by a time constraint $\alpha(\geq 0)$. Thus, in the case including a time constraint $(\alpha>0)$, the larger the number of neighbors of player $i$, the more the schedule adjustment and the opportunity cost seem to increase for $i$, so $c\left(d_{i}\right)$ increases with respect to the degree $d_{i}$ of $i$. This case includes, for example, a situation in which people face a lot of difficulty to make a new friend in a community due to the shortage of free time. In the case without a time constraint $(\alpha=0)$, the number of neighbors of $i$ does not affect the schedule adjustment or the opportunity cost for $i$ at all, so $c\left(d_{i}\right)$ remains constant with respect to the degree $d_{i}$ of $i\left(c\left(d_{i}\right)=\bar{c}\right)$. The fixed probability $p_{i j}$ for the first step in the dynamic process is uniformly distributed. The initial network $g^{1}$ is the empty network, namely, the network where every player has no links, since we are interested in a network growth in a community. The final period of the dynamics is denoted by $T$.

We run a Monte Carlo simulation with 1,000 iterations, and examine its mean in the analysis.

\subsection{Results}

Let us examine two cases for the communication cost $c\left(d_{i}\right)$ : the case that $c\left(d_{i}\right)$ is constant and the case that $c\left(d_{i}\right)$ increases with respect to the degree of $i$. We will analyze the dynamics of the network from the viewpoint of the network density (ND), the average clustering coefficient (ACC), the number of isolated players, and the standard deviation of each player's degree.

The network density (ND) is the ratio of the number of links present to the maximum number possible, and is calculated as follows:

$$
\mathrm{ND}=\frac{\sum_{i} d_{i}}{2 \cdot\left(\begin{array}{l}
n \\
2
\end{array}\right)}
$$

The relation in which the neighbors of a player are connected to each other, namely the triangle in the network, is called "transitivity" or "clustering" (e.g. Jackson 2008; Newman 2010). The measure that looks at all pairs of players that are linked to $i$ and then considers how many of them are linked to one another is called as the individual clustering coefficient $C l_{i}(g)$. The average of the individual 


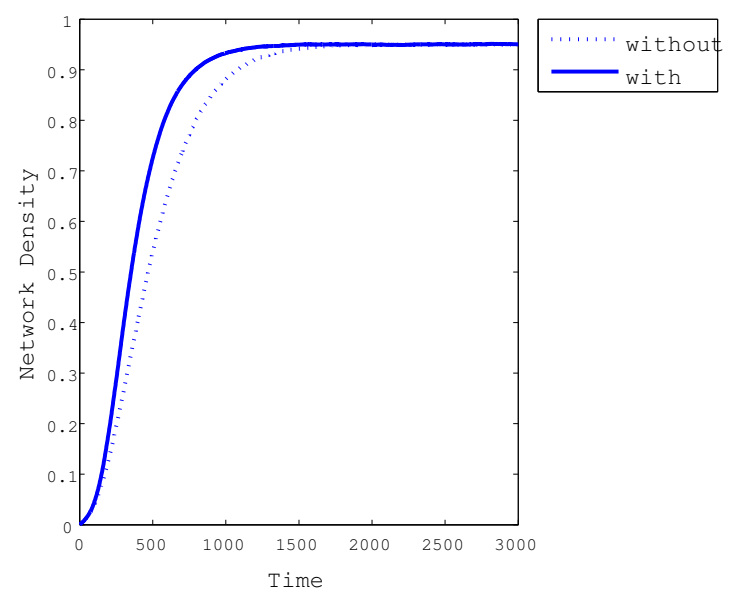

Fig. 1 Network density in the case with $\alpha=0$

clustering coefficients of all players is the average clustering coefficient (ACC), and is defined as follows:

$$
\mathrm{ACC}=\frac{\sum_{i} C l_{i}(g)}{n},
$$

where

$$
C l_{i}(g)=\frac{\#\left\{j k \in g \mid k \neq j, j \in N_{i}(g), k \in N_{i}(g)\right\}}{\#\left\{j k \mid k \neq j, j \in N_{i}(g), k \in N_{i}(g)\right\}} .
$$

Among many conceptualizations of social capital, some of the literature on social network theory view the clustering coefficient and the network density as measures of social capital (e.g., Coleman 1988; Borgatti et al. 1998). For example, Coleman (1988) reports that higher clustering prevents a person from betraying others because his/her behavior is monitored by a third person who is their common friend. Moreover, Borgatti et al. (1998) write that higher density provides a basis for sharing knowledge that can finally create synergetic effects in collaboration of persons with different backgrounds. Focusing on these aspects of the broad concept of social capital, the clustering coefficient and the network density have been applied as its indices.

\subsubsection{The Case Where Communication Cost $c\left(d_{i}\right)$ Remains Constant with Respect to the Degree $d_{i}$ of $i$ : The Case of $\alpha=0$}

Here, let us consider the case where $c\left(d_{i}\right)$ is constant; namely, $\alpha=0$. Figures 1-4 show the dynamics of the network density, average clustering coefficient, number of isolated players, and standard deviation of each player's degree, respectively. In each figure, a dotted line represents the case without disasters, while a solid line represents the case with disasters. 


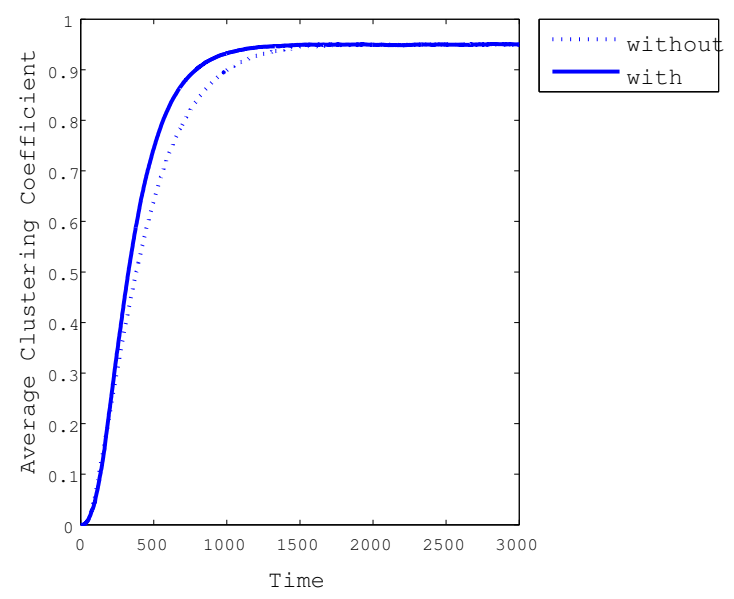

Fig. 2 Average clustering coefficient in the case with $\alpha=0$

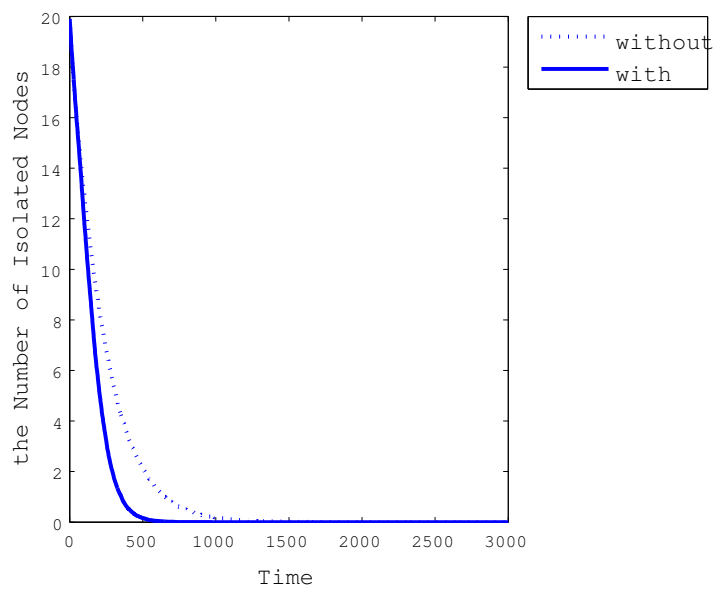

Fig. 3 The number of isolated players in the case with $\alpha=0$

By comparing these two cases, we can see that every index converges to almost the same level in the long-term. However, we also observe that the paths to the convergent state are not the same. In the case with disasters, the network density and average clustering coefficient increase quickly, and isolated players disappear quickly. The standard deviation of the degree quickly achieves a maximum, and its maximum is smaller than that in the case without disasters, which means that in the case including disasters there exists less disparity in degrees between players. Therefore, in the case of a constant communication cost, it is demonstrated that the link formation motivated by altruism during disasters has the effect of accelerating convergence speed. In the following, let us call this effect a "speed up effect." 


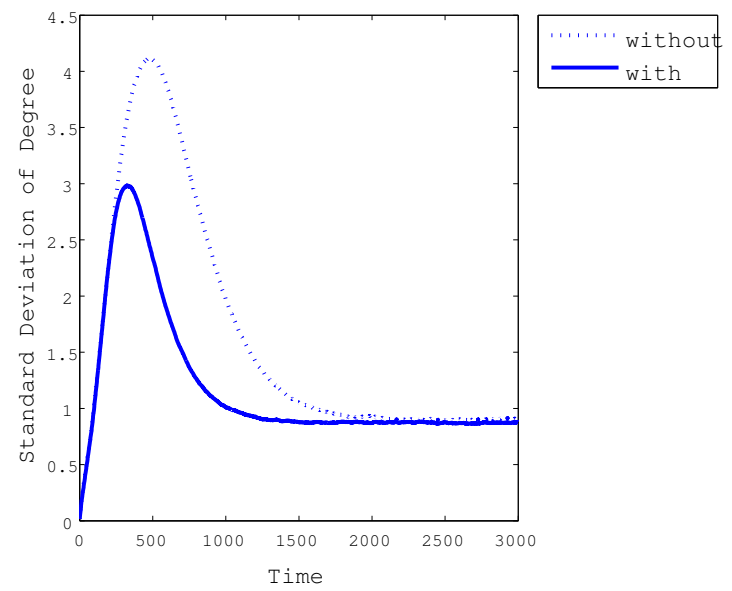

Fig. 4 Standard deviation of degree in the case with $\alpha=0$

Let us consider the reason for the above difference. Under our present settings, the communication cost is constant, and $\bar{c}=0.2$. Then, in a time of peace player $i$ forms a link with player $j$ if $j$ has at least one link other than $i$. Conversely, $i$ does not form a link with player $j$ if $j$ is isolated. Therefore, $i$ and $j$ form link $i j$ if $i$ and $j$ have links $i k$ and $j l$, respectively $(k \neq j, l \neq i)$. Consequently, in a time of peace, every pair $i j(i j \notin g)$ that adds link $i j$ can be depicted with respect to $d_{i}$ and $d_{j}$ as shown in Figure 5.

Therefore, in the case without disasters, the trigger allowing an isolated player to obtain an initial link can only be an error/mutation. Once an isolated player forms a link, then the player autonomously forms links with others having at least one link with players other than her/him. If every player has at least one link formed by error/mutation, then all players start adding links autonomously, which leads a network to converge to a state close to a complete network (a network $g$ in which $d_{i}=n-1, \forall i$ ) in the long-term.

On the other hand, in the case that includes disasters, the player $i$ with the larger degree from pair $i j$ has an altruistic preference during a disaster. Therefore, the more likely $j$ is to be isolated, the more $i$ prefers to link with $j$. Consequently, every pair $i j(i j \notin g)$ that adds the link $i j$ can be depicted with respect to $d_{i}$ and $d_{j}$ as shown in Figure 6. Unlike Figure 5, this figure shows that players with $d_{j}=0$ can form a link, while players with $d_{j}=19$ cannot form a link. Therefore, not only error/mutation in times of peace but also rational decision making during disasters enables isolated players to obtain an initial link, allowing isolated players to eliminated sooner. Once every player has an initial link, players start adding links autonomously, which leads the network to converge quickly to a state close to a complete network. Moreover, after player $i$ adds the link $i j$ during a disaster, $i$ deletes the link $i k(k \neq j)$, that is, a link which she/he has with the player $k$ having the largest degree among the set of $i$ 's neighbors $N_{i}(g)$. Consequently, this process increases player $j$ 's degree and decreases $k$ 's degree, so it is demonstrated that in the case with disasters, the standard deviation of the degree decreases. 


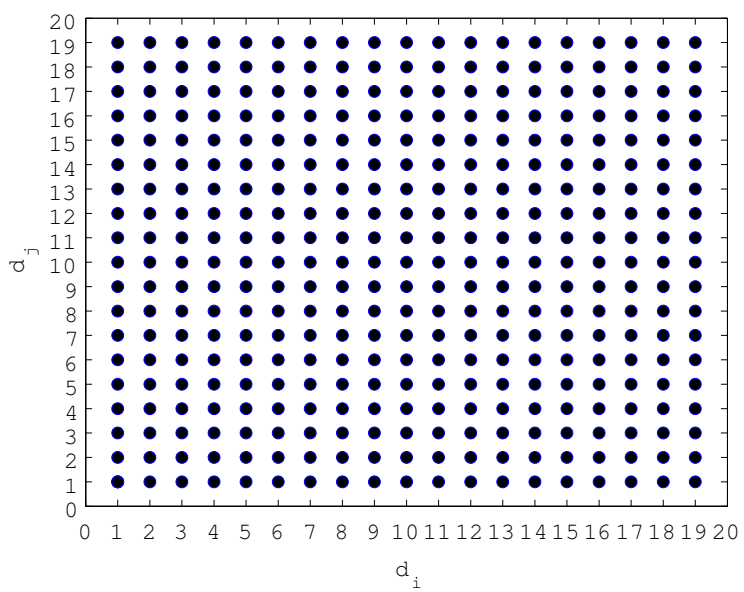

Fig. 5 Every pair who adds link $i j$ in time of peace in the case with $\alpha=0$

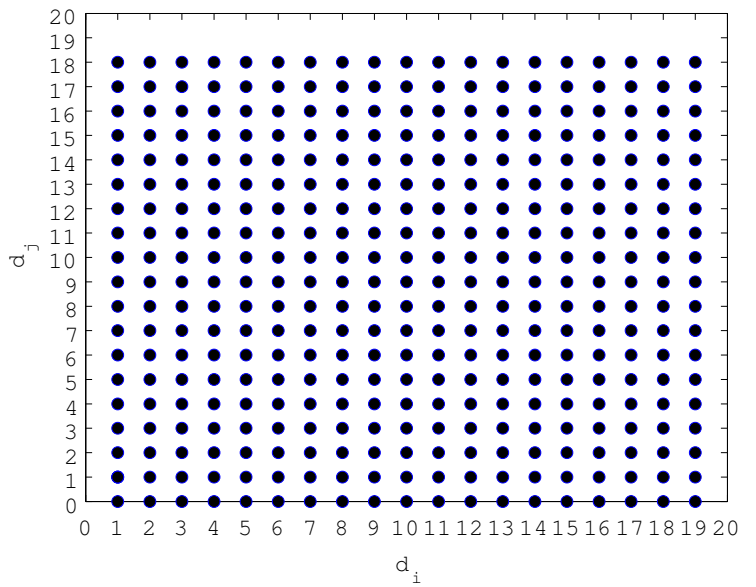

Fig. 6 Every pair who adds link $i j$ during disasters in the case with $\alpha=0$

4.2.2 The Case Where Communication Cost $c\left(d_{i}\right)$ Increases with Respect to the Degree $d_{i}$ of $i$ : The Case of $\alpha=1$

Here, we consider the case where $c\left(d_{i}\right)$ increases with respect to $d_{i}$; namely $\alpha>0$. We assume that $\alpha=1$. Figures 7-10 show the dynamics of the network density, average clustering coefficient, number of isolated players, and standard deviation of each player's degree, respectively. In each figure, a dotted line represents the case without disasters, while a solid line represents the case with disasters.

First, let us focus on the case without disasters, and examine the effect of the change of $\alpha$ from $\alpha=0$ to $\alpha=1$ on the dynamics of network. By comparing these 


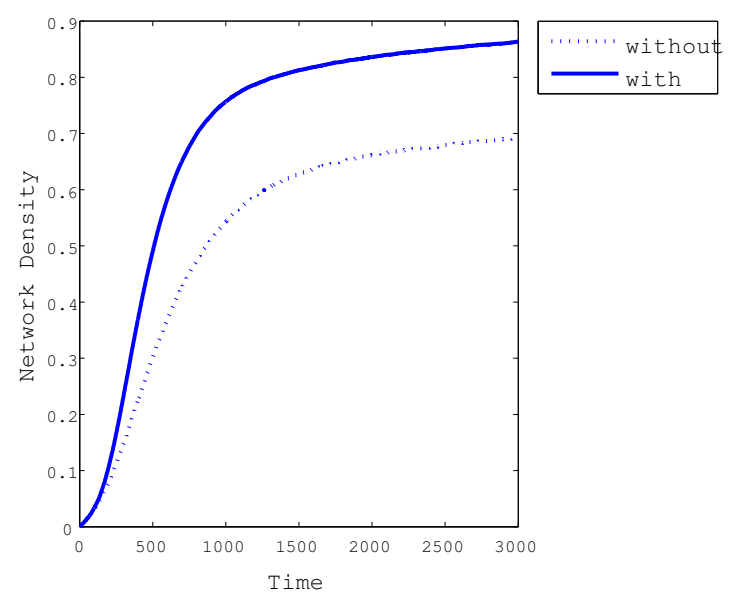

Fig. 7 Network density in the case with $\alpha=1$

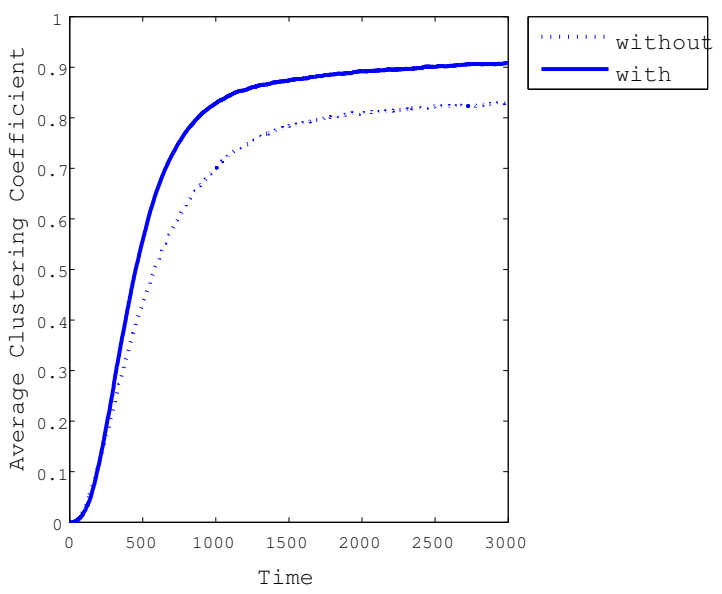

Fig. 8 Average clustering coefficient in the case with $\alpha=1$

two cases, we can observe that in the case with $\alpha=1$ isolated players do not necessarily disappear, even in the long run, unlike in the case with $\alpha=0$. In the case with $\alpha=1$, the standard deviation of the degree does not decrease, and maintains a higher level. Therefore, it is demonstrated that the network never attains the state of an almost complete network, but instead converges to a relatively sparse network.

Let us consider the reason for the phenomenon described above. In the case that the communication cost increases with respect to the degree, the marginal cost of forming a link is larger for player $i$ that have a larger degree $d_{i}$. Therefore, if player $j$ 's degree is smaller, then $i$ cannot gain additional net utility from adding 


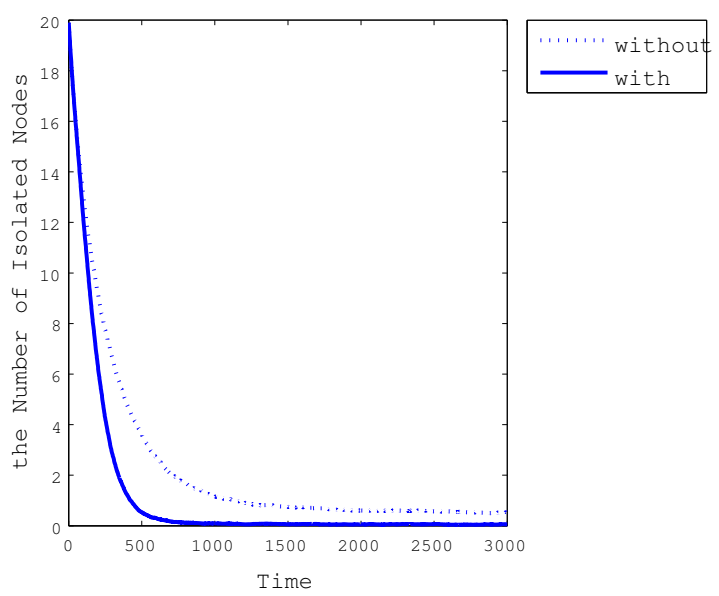

Fig. 9 The number of isolated players in the case with $\alpha=1$

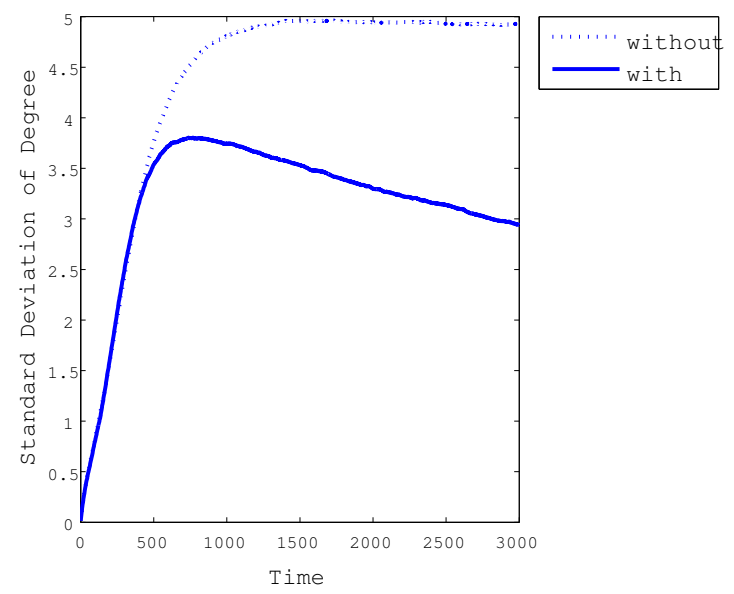

Fig. 10 Standard deviation of degree in the case with $\alpha=1$

a link with $j$. This means player $i$ is less likely to form a link with the player $j$. Consequently, we can represent every pair $i j(i j \notin g)$ that adds the link ij in a time of peace with respect to $d_{i}$ and $d_{j}$ as depicted in Figure 11. The figure for the case with $\alpha=1$, unlike that for the case with $\alpha=0$, shows that players whose degrees are larger tend to connect only with others whose degrees are also large. Thus, isolated players and players who have smaller degrees cannot increase their degrees, so the standard deviation of the degree remains large, and the network density and average clustering coefficient remain small.

Next, on the basis of the above consideration, let us examine the dynamic effect of the altruistic preference on the network formation when $\alpha=1$, and compare the 
case with disasters with the one without disasters. By comparing these two cases, we see that the standard deviation of the degree in the case with disasters reaches a lower maximum of around $t=700$, and that it then decreases after reaching this maximum. Moreover, we can see that isolated players are eliminated, and the network density and average clustering coefficient show higher levels after around $t=1000$. Although the level is not as high as that in the case with $\alpha=0$, the shape of the paths is almost the same. If we compare the network in the case with disasters with that in the case without disasters, we can observe that the network in the case with disasters is more likely to converge to an almost complete network. Therefore, the link formation motivated by altruistic preferences during disasters in the case with $\alpha=1$ has the effect of accelerating the convergence speed (the "speed up effect"), and also increases the level of convergence. In the following, we will call the latter effect the "level effect." Then, the "level effect" is an additional dynamic effect of altruistic preferences during disasters on the network formation, in the case with $\alpha=1$.

Let us consider the reason for the above difference. When the player $i$ with the larger degree from pair $i j$ has an altruistic preference during disasters, we can represent every pair $i j(i j \notin g)$ that adds link $i j$ with respect to $d_{i}$ and $d_{j}$ as depicted in Figure 12. As we can observe from the figure, a player $i$ with a larger degree obtains an altruistic preference during disasters, so the smaller $j$ 's degree is, the more likely $i$ is to link with $j$. Through this process, players who have small degrees in times of peace can increase their degrees during disasters. Moreover, the increase in the degree $d_{j}$ also enables $j$ to connect with other players in time of peace who have large degrees, which accelerates the autonomous link formation among players. Owing to the above reasons, the network becomes denser in the case with $\alpha=1$, and the "level effect" can be observed. Similarly to the case of $\alpha=0$, after link $i j$ is added by the altruistic preference during disasters, player $i$ then deletes the link $i k(k \neq j)$, that is, a link which she/he has with the player $k$ having the largest degree among the set of $i$ 's neighbors $N_{i}(g)$. Consequently, this process increases the degree of player $j$ and decreases that of $k$, so it is demonstrated that in the case including disasters, the standard deviation of the degree decreases.

\section{Network Formation Including Disasters Affecting Multiple Pairs}

The previous section considered the situation in which only one pair of players has a chance to form a link based on altruistic preference when a disaster occurs. However, a larger-scale disaster affects more people. Therefore, this section considers a situation in which more pairs of players form links based on altruistic preferences when a larger-scale disaster occurs, and we thus analyze the dynamic effect of the scale of disasters on social networks and social welfare. Note that this model assumes that the occurrence of disasters is independent of social network structure, while it has been pointed out that, in reality, social vulnerability depends on social network structure (e.g., Nakagawa and Shaw 2004; Murphy 2007). Although it is an important aspect, since our focus is to examine the impact of temporal altruistic behaviors on the network formation process that is more clearly described with a simpler framework, the endogenous vulnerability will be the topic of future research. 


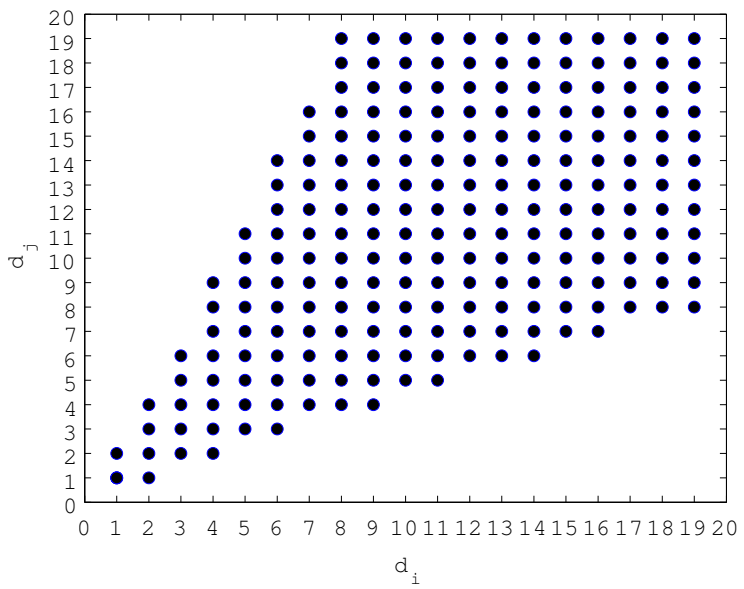

Fig. 11 Every pair who adds link $i j$ in time of peace in the case with $\alpha=1$

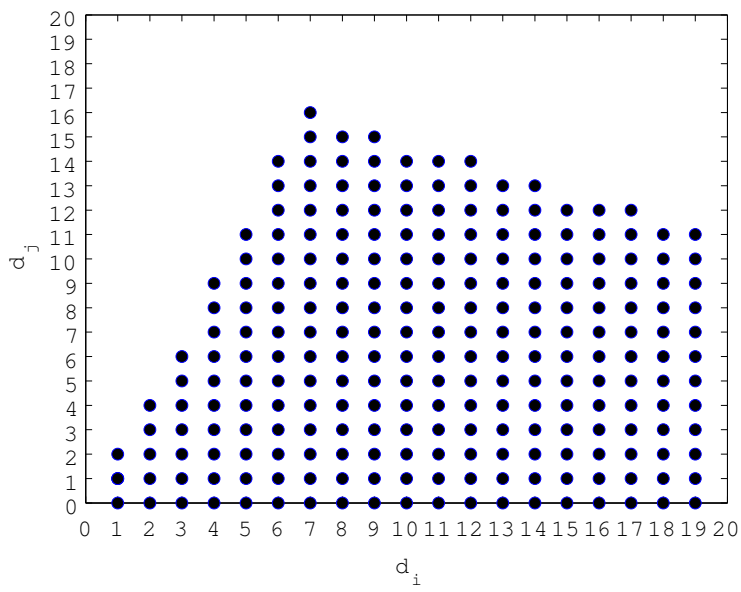

Fig. 12 Every pair who adds link $i j$ during disasters in the case with $\alpha=1$

\subsection{Extension of the Dynamic Process}

This section extends the fifth step of the dynamics shown in the previous section in order to model a situation in which $M$ pairs form links based on altruistic preferences during a disaster, assuming that $M$ becomes larger as the scale of the disaster grows. More specifically, if $M \geq 2$, we execute step 5-1 shown below after network $g_{2}^{t}$ is formed in the fifth step.

$5-1$

A pair of players $i^{\prime} j^{\prime}$ is randomly identified with fixed probability distribution $\left\{p_{i^{\prime} j^{\prime}}\right\}$ where $p_{i^{\prime} j^{\prime}}>0$ for each $i^{\prime} j^{\prime}$, and then pair $i^{\prime} j^{\prime}$ decides whether or not 
to add link $i^{\prime} j^{\prime}$ as shown in the fifth step. The above process is repeated $M-1$ times and network $g_{3}^{t}$ is created.

Network $g_{3}^{t}$ is expressed as $g^{t+1}$ at the beginning of period $t+1$. More specifically, network $g_{3}^{t}$ is obtained as follows.

After process (A) in the fifth step, let $g_{2,1}^{t}:=g_{2}^{t}$, and if $M \geq 2$, iterate as follows.

For $m=1,2, \cdots, M-1$, given $g_{2, m}^{t}$, identify players $i^{\prime}$ and $j^{\prime}$ with probability $p_{i^{\prime} j^{\prime}}$.

- In case $d_{i^{\prime}} \neq d_{j^{\prime}}$

Redefine players $i^{\prime}$ and $j^{\prime}$ such that their degrees satisfy $d_{i^{\prime}}>d_{j^{\prime}}$. Moreover, define another player $k^{\prime}$ in the set of the neighbors of player $i^{\prime}$ such that $d_{k^{\prime}} \geq d_{l^{\prime}}, \forall l^{\prime} \in N_{i^{\prime}}\left(g_{2, m}^{t}\right)$. If more than one player satisfies the condition to be player $k^{\prime}$, select one randomly from among them, and define that player as $k^{\prime}$.

- In case $i^{\prime} j^{\prime} \notin g_{2, m}^{t}$ If $u_{i^{\prime}}^{a}\left(g_{2, m}^{t}+i^{\prime} j^{\prime}\right) \geq u_{i^{\prime}}^{a}\left(g_{2, m}^{t}\right)$ and $u_{j^{\prime}}^{s}\left(g_{2, m}^{t}+i^{\prime} j^{\prime}\right) \geq u_{j^{\prime}}^{s}\left(g_{2, m}^{t}\right)$ with one inequality strict, then $g_{2, m+1}^{t}=g_{2, m}^{t}+i^{\prime} j^{\prime}-i^{\prime} k^{\prime}$ with probability $(1-\epsilon)$ and $g_{2, m+1}^{t}=g_{2, m}^{t}$ with probability $\epsilon$. Otherwise, $g_{2, m+1}^{t}=g_{2, m}^{t}$ with probability $(1-\epsilon)$ and $g_{2, m+1}^{t}=g_{2, m}^{t}+i^{\prime} j^{\prime}-i^{\prime} k^{\prime}$ with probability $\epsilon$.

- In case $i^{\prime} j^{\prime} \in g_{2, m}^{t}$ $g_{2, m+1}^{t}=g_{2, m}^{t}$.

- In case $d_{i^{\prime}}=d_{j^{\prime}}$ $g_{2, m+1}^{t}=g_{2, m}^{t}$.

\section{End}

$g_{3}^{t}=g_{2, M}^{t}$.

\subsection{Functions and Parameters}

Let us continue to use the same parameters and functions shown in equations (2)(7) for this section. We specify probability $p_{i^{\prime} j^{\prime}}$ with which pair $i^{\prime} j^{\prime}$ is identified for step 5-1 as uniform distribution $p_{i^{\prime} j^{\prime}}=1 /\left(\begin{array}{c}n \\ 2\end{array}\right)$. In addition, let us focus on the case where communication cost $c\left(d_{i}\right)$ increases with respect to degree $d_{i}$, namely, the case under time constraint $\alpha=1$.

The targeted society is assumed to have a large standard deviation of the degree for an initial period ${ }^{1}$. One of the reasons for realizing such a society is that it has not experienced any disasters before. Therefore, given an initial network, that is, a network that has not experienced any disasters before ${ }^{2}$, this section

1 This is because even if $M$ is so large, if a network has a small standard deviation of the degree, it is more likely that $d_{i^{\prime}}=d_{j^{\prime}}$ for pair $i^{\prime} j^{\prime}$ in step 5-1, and consequently, we are less likely to observe $M$ number of link formations with altruistic preferences. This hampers our analysis of the true effect of the difference in $M$.

2 This section creates a network with a large standard deviation, running a process without any disaster as in Section 3. 2.2 for $1 \leq t \leq 1500$. Then, we define the network at $t=1501$ as an initial network for this section, and analyze the situation with the disaster occurring after this period. Figure 10 clearly shows that the network around $t=1500$ has a large standard deviation of degree. 


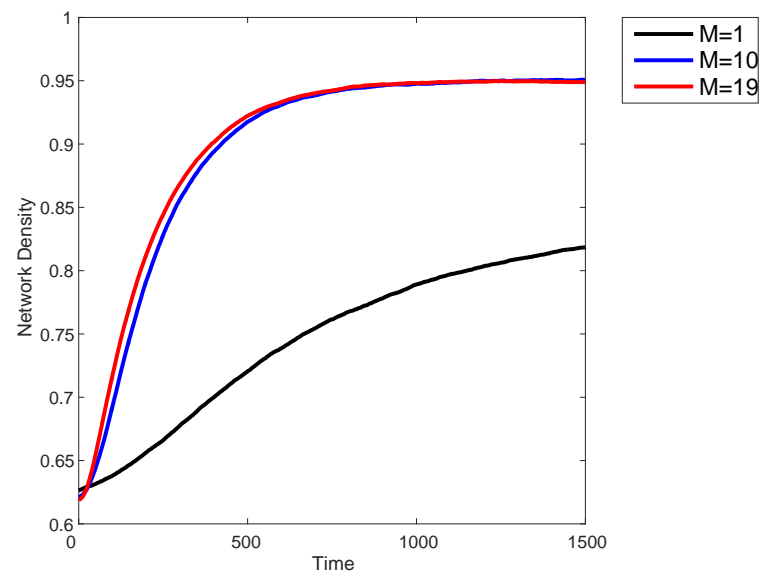

Fig. 13 Comparative dynamics of network density with respect to $M$

considers the situation in which the extension of the fifth step is executed during a disaster, and investigates how $M$ pairs of link formations with altruistic preferences affect the network density, standard deviation of degree, and social welfare. We measure social welfare in each period by aggregating each player's utility with selfish preference at the end of the period. More specifically, social welfare (SW) is calculated as follows:

$$
\mathrm{SW}=\sum_{i=1}^{n} u_{i}^{s}\left(g^{t}\right)
$$

Disasters are rare events that do not occur in every period; thus, every individual spends a much longer time without experiencing any disasters, which lets us measure the social welfare with selfish rather than altruistic preferences. Similar to the previous section, we run a Monte Carlo simulation with 1,000 iterations and examine its mean in the analysis.

\subsection{The Scale of Disasters and Its Dynamic Effect}

Let us consider three different cases according to the scale of the disaster, namely, when the number of pairs forming links in the fifth step are 1) only one $(M=1)$, 2) five percent of all possible pairs $\left(M=10\left(\simeq 0.05 \cdot\left(\begin{array}{c}n \\ 2\end{array}\right)\right)\right)$, and 3) ten percent of all possible pairs $\left(M=19\left(=0.10 \cdot\left(\begin{array}{l}n \\ 2\end{array}\right)\right)\right)$. Case $M=1$ corresponds with the case in Section 3. 2. 2, which considers the occurrence of disasters. Figures 13-15 show the dynamics of the network density, standard deviation of each player's degree, and social welfare, respectively, according to the scale of the disaster. Figure 16 shows the enlarged view during $1 \leq t \leq 150$ in Figure 15. In each figure, the black, blue, and red lines represent the cases $M=1, M=10$, and $M=19$, respectively.

We observe from Figures 13-14 that as $M$ becomes larger, the standard deviation of degree becomes smaller, and the network density becomes larger more 


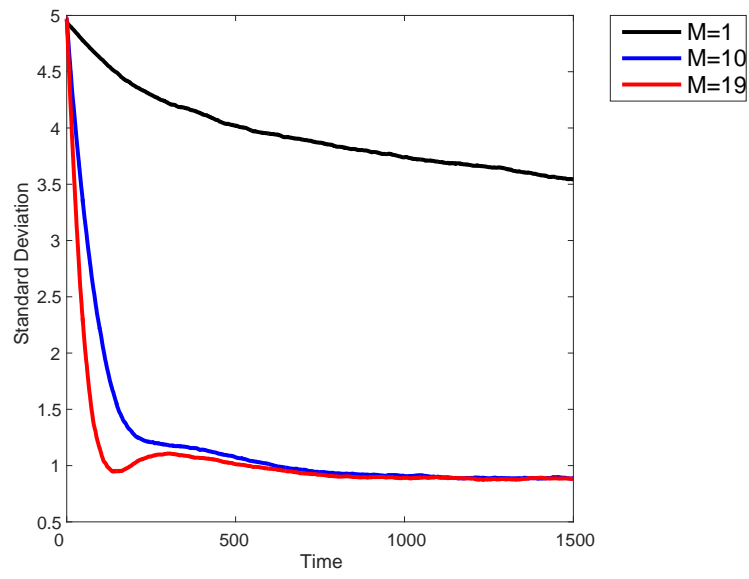

Fig. 14 Comparative dynamics of standard deviation of degree with respect to $M$

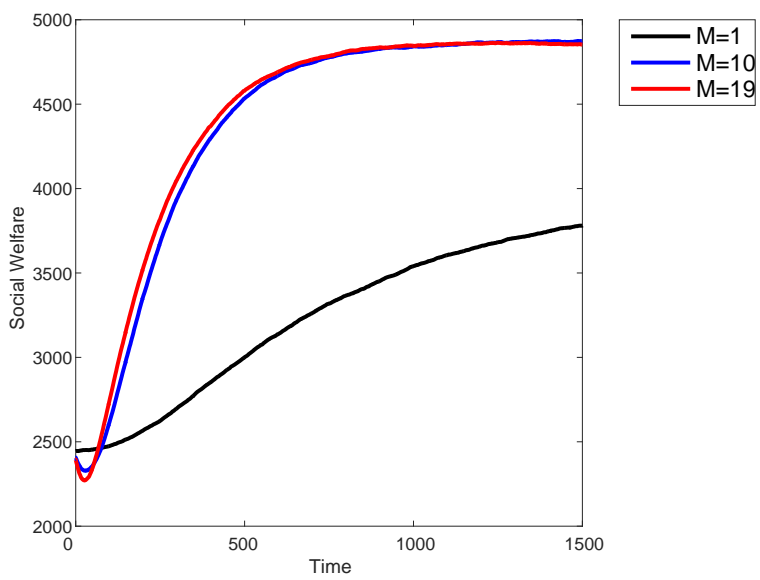

Fig. 15 Comparative dynamics of social welfare with respect to $M$

quickly, finally converging to a steady state. Moreover, Figure 16 shows that after $t \geq 80$, as $M$ becomes larger, social welfare increases, finally converging to a steady state as well. Let us consider the reason for the above phenomena. As $M$ becomes larger, more links among players are redistributed during a disaster, which equalizes the degree between players and decreases the standard deviation of degree. Consequently, every player is more likely to have an equal chance to interact with each other, which accelerates autonomous link formation during a time of peace. In other words, the dynamic positive externality, which is generated by link formation motivated by altruistic preference, continues even after a disaster, and accelerates the realization of a dense network and improved social welfare. 


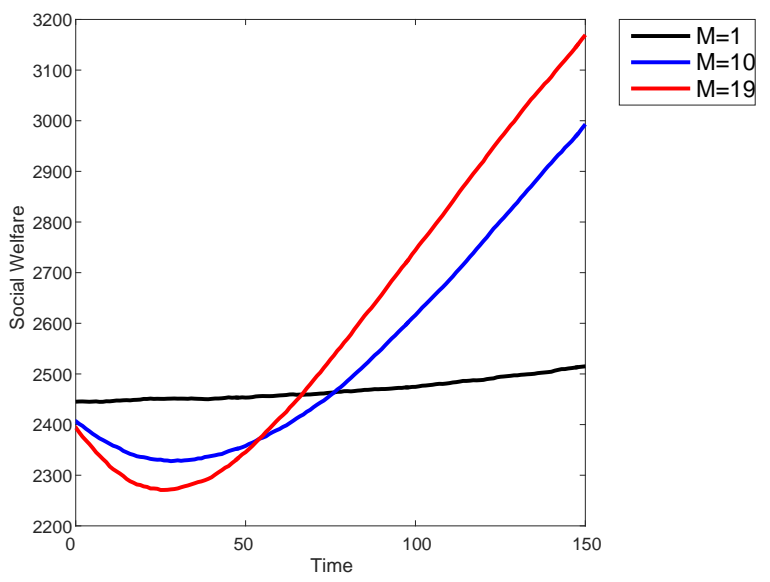

Fig. 16 Enlarged view of Figure 15 during $1 \leq t \leq 150$
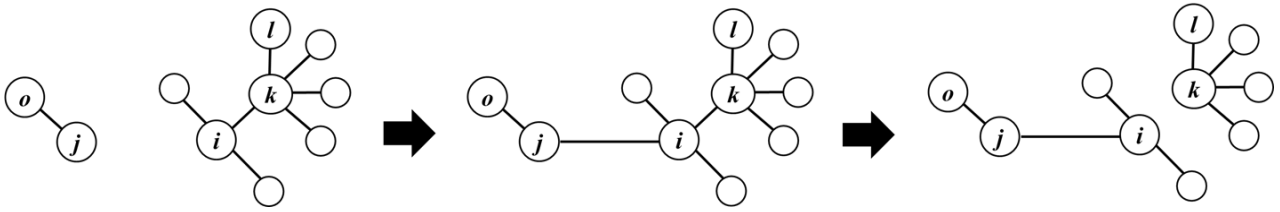

Fig. 17 Dynamics process in which player $i$ with altruistic preference forms link $i j$

However, as $M$ becomes larger, social welfare becomes smaller during $1 \leq t<$ 80 as Figure 16 indicates. The above finding can be explained analytically. Let us rewrite social welfare using standard deviation $\sigma_{d}$ of degree and average degree $\mu_{d}$ as follows:

$$
\mathrm{SW}=n\left(0.8 \cdot\left(\sigma_{d}^{2}+\mu_{d}^{2}\right)-\mu_{d}\right) .
$$

The proof of equation (12) appears in the appendix B. As mentioned previously, as $M$ increases, more links among players are redistributed during a disaster, which leads to a decrease in the standard deviation of degree. In other words, only standard deviation $\sigma_{d}$ of degree decreases, leaving average degree $\mu_{d}$ constant. According to equation (12), $\frac{\partial \mathrm{SW}}{\partial \sigma_{d}}>0$, and thus, other things being equal, as $\sigma_{d}$ decreases, so does SW.

To further analyze why the larger decrease occurs temporally in social welfare, let us focus on the change in each player's utility. We see that when pair $i j$ forms a link motivated by an altruistic preference on a network with large standard deviation of degree, a decrease of utilities with selfish preference of each 1) player $i$, 2) player $k$ who is $i$ 's neighbor, and 3) players $l$ who are $k$ 's neighbors is more likely to occur, which results in a decrease in social welfare. We explain the above statement by illustrating a typical case depicted on the left-hand side of Figure 17 , in which pair $i j$ forms a link with an altruistic preference in a network that is 
formed autonomously. As depicted in the figure, we assume that 1) $1<d_{i}<d_{k}$ and that 2) a component that $j$ belongs to and a component that $i, k$, and $l$ belong to are both distinct. If player $i$ with an altruistic preference forms link $i j$ with player $j$ whose degree satisfies $d_{i}>d_{j}$ (see the middle of Figure 17), $i$ deletes link $i k$ with player $k$ who has the largest degree among $i$ 's neighbors (see the right-hand side of Figure 17). Through this process, 1) a change in $i$ 's utility with selfish preference is denoted as follows:

$$
\begin{aligned}
& u_{i}^{s}(g+i j-i k)-u_{i}^{s}(g) \\
= & \left(d_{j}-1\right)-\left(d_{k}-1\right) \\
= & d_{j}-d_{k}<0 . \quad\left(\because d_{j}<d_{i}<d_{k}\right)
\end{aligned}
$$

Hence, $i$ 's utility with selfish preference necessarily decreases. 2) Regarding a network that has been formed autonomously, $k$ obtains a net benefit from connecting with $i$. Thus, $k$ 's utility necessarily decreases if $k$ loses link $i k$. A change in $k$ 's utility is denoted as follows:

$$
\begin{aligned}
& u_{k}^{s}(g+i j-i k)-u_{k}^{s}(g) \\
= & -\left(d_{i}-1\right)-\bar{c} \cdot\left(\left(d_{k}-1\right)^{\alpha+1}-d_{k}^{\alpha+1}\right) \\
= & 0.4 \cdot d_{k}-d_{i}+0.8<0 . \\
& (\because \text { network } g \text { is autonomously created. })
\end{aligned}
$$

3) Since $k$ loses link $i k$, a change in the utility of $l$, who is one of $k$ 's neighbors, is denoted as follows:

$$
\begin{aligned}
& u_{l}^{s}(g+i j-i k)-u_{l}^{s}(g) \\
= & \left(d_{k}-1-1\right)-\left(d_{k}-1\right) \\
= & -1<0 .
\end{aligned}
$$

Hence, $l$ 's utility necessarily decreases, which means that negative externality to $l$ exists. As the standard deviation of degree becomes larger, degree $d_{k}$, namely, the number of neighbors $\left(=d_{k}\right)$, is likely to increase, and therefore, more neighbors of $k$ face decreases in utility; that is to say, the formation of link $i j$ generates more negative externalities.

In addition to the above players' utilities, the utilities of player $j$ and $j$ 's neighbor $o$ are also altered. Since $j$ obtains link $i j$, each change in their utilities is denoted as follows:

$$
\begin{aligned}
& u_{j}^{s}(g+i j-i k)-u_{j}^{s}(g) \\
= & \left(d_{i}-1\right)-\bar{c} \cdot\left(\left(d_{j}+1\right)^{\alpha+1}-d_{j}^{\alpha+1}\right) \\
= & d_{i}-0.4 \cdot d_{j}-1.2>0 .\left(\because 1<d_{i} \text { and } d_{j}<d_{i}\right) \\
& u_{o}^{s}(g+i j-i k)-u_{o}^{s}(g) \\
= & d_{j}-\left(d_{j}-1\right) \\
= & 1>0 .
\end{aligned}
$$

Hence, the utilities of player $j$ and $o$ necessarily increase. 
If we denote a change in social welfare by $\Delta(\mathrm{SW})$, we can derive $\Delta(\mathrm{SW})$ as follows, considering equations (13)-(17) and the presence of a number $d_{k}$ of $k$ 's neighbors $l$ and a number $d_{j}$ of $j$ 's neighbors $o$ :

$$
\begin{aligned}
& \Delta(\mathrm{SW}) \\
= & \left(d_{j}-d_{k}\right)+\left(0.4 \cdot d_{k}-d_{i}+0.8\right) \\
& +(-1) \cdot d_{k}+\left(d_{i}-0.4 \cdot d_{j}-1.2\right)+1 \cdot d_{j} \\
= & -1.6 \cdot\left(d_{k}-d_{j}\right)-0.4<0 .\left(\because d_{j}<d_{i}<d_{k}\right)
\end{aligned}
$$

In conclusion, for the case depicted in Figure 17, in which a link is formed with an altruistic preference, the negative effects of 1)-3) dominate, and therefore, social welfare decreases. Furthermore, as $M$ becomes larger, the above-mentioned negative effects increase, and as a result, social welfare decreases more significantly.

\section{Discussion}

Finally, let us consider the implications of the results of our model for "a paradise built in hell." As previously mentioned, the situation where the communication cost of interacting with a person increases with respect to the degree, that is, where the additional cost in opportunities of interacting with a new friend increases as a player's number of friends is larger, can include cases where it is not easy to adjust one's schedule for meeting friends or where the opportunity cost of interacting in a community is high owing to a busy job. In such situations, people tend to be selective about persons with whom they interact as their number of friends increases. More specifically, people tend to choose to interact with others who bring benefits that outweigh the high costs. Because the interaction requires mutual consent, this results in the "disparity in relationships." That is, a disparity between those who have many relationships and those who have few relationships can be created. This result can be seen from Figure 10, which shows a monotonic increase in the standard deviation of each player's degree. Furthermore, those who have more relationships are more likely to collect the latest information, and to have a more favorable opportunity to make decisions related with business or politics through cooperation with their friends. Consequently, this would strengthen the social structure where the "rich in relationships" obtain better chances.

In contrast, disasters induce a temporary altruism factor, which enables the "poor in relationships" to become integrated into large human networks. Once they are integrated, they then begin to increase their number of relationships even in regular life. Consequently, society as a whole increases its network density and average clustering coefficient, which are regarded as indices of social capital in social network theory. Therefore, the society following disasters can have an enhanced social capital and a narrower "disparity in relationships." The enhancement of social capital facilitates the sharing of information and increases the possibilities for coordinated behavior. The smaller disparity also increases the possibilities for decision making, such as decisions by majority. To summarize, the alteration of social networks caused by disasters could have the potential to modify social institutions or conventions. This phenomenon corresponds with the long-term aspect of "a paradise built in hell." In other words, a social network that is the foundation of a series of large institutional reforms following disasters would be created mainly 
through the temporary altruism that immediately follows disasters. In addition, if we regard an increase in communication cost as a phenomenon that is more often seen in regular life in urban areas, we can conclude that the qualitative changes in the shapes of social networks caused by disasters and the long-term developments of "a paradise built in hell" would be more likely to be observed in urban areas.

If the temporary altruism induced by large-scale disasters is seen among more people, the poor in such relationships are more likely to become integrated into networks constituted by the rich. Thus, the long-term effect would surely have a larger potential impact. Meanwhile, the temporary altruism during disasters encourages people to interact more with the poor than with the rich in their relationships. Thus, rich people in relationships are temporarily less likely to collect the latest information and have favorable opportunities. Moreover, since richer people in relationships have many friends, the negative effect spreads to their friends too, resulting in these friends also having fewer chances for collecting the latest information and having favorable opportunities. As the scale of the disaster enlarges, the above-mentioned temporary effect becomes more serious for the rich in relationships and their surrounding friends. In summary, as altruistic behavior immediately after disasters, namely, the short-term aspect of "a paradise built in hell," is seen more frequently owing to the larger-scale disaster, it not only generates a larger negative temporary externality to the rich in relationships and the surrounding people, it also creates a larger positive dynamic externality. Thanks to the latter externality, a network that allows for coordinated behavior and collective decision making is more likely to be formed quickly, and thus, the long-term aspect of "a paradise built in hell" is more likely to be realized.

This study exclusively focuses on the dynamic impact of altruistic behaviors that temporally emerge in the aftermath of disasters. However, there are a variety of factors that are critical for issues of the communities such as changes of community members, among which emigration is one of the most serious phenomena for sustainability of vitality of affected communities or their social capital (e.g., Drabo and Mbaye 2011). Although the model does not deal with emigration in an explicit way, it may be possible to imagine that isolated players are more likely to migrate to other regions. By applying the network density and the clustering coefficient as indices, this study examines the social capital that includes those isolated players who decrease the values of the indices. In other words, it seems less appropriate to exclude those potential emigrants from evaluation of social capital that would result in an increase in those values. On the other hand, an interesting future topic is to include the impact of in-migration of volunteer workers who may play an important role as catalytic agents in social networks.

\section{Conclusion}

Through the application of a social network formation model based on game theory, this study analyzed the long-term effects on social networks of link formation motivated by altruistic preferences during disasters. In a situation where the communication cost of maintaining a link increases, the disparity in the number of relationships was shown to become larger. In other words, people who have more (fewer) relationships to start with obtaining new relationships at a faster (slower) rate. However, if disasters occur in this situation, then the link formation that is 
motivated by the altruistic preferences leads to the integration of isolated players into a network, which also leads to an increase in their number of relationships even long after disasters. This study figured out two sorts of externalities; the redistribution generates a negative cross-sectional externality for persons who have many neighbors and the neighbors in terms of utility that results in a decrease in social welfare, while it has a dynamic positive externality that accelerates the formation of a network with a potential to lead to the long-term outcome of "a paradise built in hell." It was also pointed out that the dynamic effect is composed of "speed up effect" and a "level effect" of social network formation, and moreover, the disparity in the number of relationships among people decreases. It was also implied that if some groups that have dominant influence in collective decision making of social institutions are changed by disasters, the long-term aspect of "a paradise built in hell" is more likely to occur. Furthermore, the study also focused on the impact of a large-scale disaster, namely, simultaneous multiple link reformation with altruistic preference, which resulted in larger effect on long-term outcome of "a paradise built in hell."

We will conclude by discussing some possible extensions and directions for future research. In addition to topics that we have already described, first of all, this study adopted an algorithm that allows only one pair of players plays a linkformation game in each step, which could be extended to ones with many players that make simultaneous decision to investigate the possibility of collaboration activities in an affected area. Second, it has been noted that people tend to value relationships formed during disasters more highly. A model that accounts for distinctions in the quality of links should be proposed. Third, the supply of goods and labor to victims from persons who have them in abundance during disaster has the potential to create new leaders or relationships in a community. A network formation that is driven by those actions will be described with a model where several types of goods and resource are dealt with. Finally, in our model the emergence of the altruistic behavior was given exogenously. Some of the previous studies have focused on the endogenous emergence or dispersal of altruism (e.g., Mitteldorf and Wilson 2000; Ohtsuki et al. 2006). In future study, we will propose a hybrid type of a model that considers endogenous altruistic behaviors.

Acknowledgements This work was partly supported by JSPS KAKENHI Grant Number 15J08041 and Kyoto University Research Development Programs 2015. 


\section{Appendix A}

Table 1 List of variables in the model

Endogenous variables

$d_{i} \quad$ the degree of player $i$

$b^{h}\left(d_{j}\right) \quad$ the benefit of each player with preference $h$ obtained from neighbor $j$

$c\left(d_{i}\right) \quad$ the communication cost, which means the cost to player $i$ of interacting with

a neighbor

$u_{i}^{h}(g) \quad$ the utility of player $i$ with preference $h$ in network $g$

Exogenous variables

$n \quad$ the number of players in a community

$h \quad$ the type of preference ( $h=s$ means a selfish preference, and $h=a$ means an altruistic one.)

$\epsilon \quad$ the probability of occurrence of an error/mutation

$p_{i j} \quad$ the probability that a pair $i j$ is identified in the first step of the dynamic process

$\eta \quad$ the probability of occurrence of a disaster

$\alpha \quad$ the parameter of the communication cost function

$\bar{c} \quad$ the cost of maintaining a link without a time constraint

$T \quad$ the final period of the dynamics

$M \quad$ the number of pairs that form links based on altruistic preferences when a disaster occurs in Section 5

\section{Appendix B}

Equation (12) is derived as follows:

$$
\begin{aligned}
\mathrm{SW} & =\sum_{i=1}^{n} u_{i}^{s}\left(g^{t}\right) \\
& =\sum_{i=1}^{n}\left(\sum_{j \in N_{i}(g)} b^{s}\left(d_{j}\right)-c\left(d_{i}\right) \cdot d_{i}\right) \\
& =\sum_{i=1}^{n}\left(\sum_{j \in N_{i}(g)}\left(d_{j}-1\right)-\bar{c} \cdot d_{i}^{\alpha+1}\right)\left(\because b^{s}\left(d_{j}\right)=d_{j}-1, c\left(d_{i}\right)=\bar{c} \cdot d_{i}^{\alpha}\right) \\
& =\sum_{i=1}^{n}\left(\left(d_{i}-1\right) \cdot d_{i}-\bar{c} \cdot d_{i}^{\alpha+1}\right) \\
& \left(\because \sum_{i=1}^{n}\left(\sum_{j \in N_{i}(g)}\left(d_{j}-1\right)\right)=\sum_{i=1}^{n}\left(d_{i}-1\right) \cdot d_{i}\right.
\end{aligned}
$$

i.e., on the left-hand side of the above formulation, $\left(d_{i}-1\right)$ appears $d_{i}$ times for each $i$.)

$$
\begin{aligned}
& =\sum_{i=1}^{n}\left(0.8 \cdot d_{i}^{2}-d_{i}\right) \quad(\because \bar{c}=0.2, \alpha=1) \\
& =n\left(0.8 \cdot\left(\sigma_{d}^{2}+\mu_{d}^{2}\right)-\mu_{d}\right) .
\end{aligned}
$$




\section{References}

1. Atsumi T (2014) Relaying support in disaster- affected areas: the social implications of a 'pay- it- forward' network. Disasters, 38(s2): s144-s156. doi:10.1111/disa.12067

2. Barton AH (1969) Communities in disaster: A sociological analysis of collective stress situations. Garden City, NY: Doubleday

3. Borgatti SP, Jones C, Everett MG (1998) Network measures of social capital. Connections, 21(2):27-36

4. Chasparis GC, Shamma JS (2013) Network formation: neighborhood structures, establishment costs, and distributed learning. Cybernetics, IEEE Transactions on, 43(6):1950-1962. doi:10.1109/TSMCB.2012.2236553

5. Coleman JS (1988) Social capital in the creation of human capital. American Journal of Sociology, 94:S95-S120

6. Drabo A, Mbaye L (2011) Climate change, natural disasters and migration: An empirical analysis in developing countries. IZA Discussion Paper, No. 5927

7. Fogleman CW, Parenton VJ (1959) Disaster and aftermath: Selected aspects of individual and group behavior in critical situations. Social Forces, 38(2):129-135. doi:10.2307/2573932

8. Fujimura M (2001) Contemporary Sociological Contrasts between Ordinary and Exceptional Times. In: Shimane K, Fujimura M (ed) Cultural Apparatus as Determiners for Exceptional Times, Hokuju Shuppan, pp 16-37 (in Japanese)

9. Giel R (1990) Psychosocial processes in disasters. International Journal of Mental Health, 19(1):7-20

10. Jackson MO, Watts A (2002) The evolution of social and economic networks. Journal of Economic Theory, 106(2):265-295. doi:10.1006/jeth.2001.2903

11. Jackson MO, Watts A (2002) On the formation of interaction networks in social coordination games. Games and Economic Behavior, 41(2):265-291. doi:10.1016/S08998256(02)00504-3

12. Jackson MO (2008) Social and economic networks. Princeton University Press, New Jersey

13. Kaniasty K, Norris FH (2004) Social support in the aftermath of disasters, catastrophes, and acts of terrorism: Altruistic, overwhelmed, uncertain, antagonistic, and patriotic communities. Bioterrorism: Psychological and Public Health Interventions, 3:200-229

14. Kotani H, Yokomatsu M (2015) Role of Local Festivals on Network Formation among a Variety of Residents in a Community. Systems, Man and Cybernetics (SMC), 2015 IEEE International Conference on. IEEE:832-839. doi:10.1109/SMC.2015.154

15. Kotani H, Yokomatsu M (2015) A Role of Ennichi and Jizobon in Expanding Interaction in a Local Community- Results of a Social Network Survey in Nagata Ward, Kobe City. Reports of the City Planning Institute of Japan, 14(May):91-98 (in Japanese)

16. Messias DKH, Barrington C, Lacy E (2012) Latino social network dynamics and the Hurricane Katrina disaster. Disasters, 36(1):101-121. doi:10.1111/j.1467-7717.2011.01243.x

17. Mitteldorf J, Wilson DS (2000) Population viscosity and the evolution of altruism. Journal of Theoretical Biology, 204(4):481-496. doi:10.1006/jtbi.2000.2007

18. Murphy BL (2007) Locating social capital in resilient community-level emergency management. Natural Hazards, 41(2):297-315. doi:10.1007/s11069-006-9037-6

19. Nakagawa Y, Shaw R (2004) Social capital: A missing link to disaster recovery. International Journal of Mass Emergencies and Disasters, 22(1):5-34

20. Newman M (2010) Networks: an introduction. Oxford University Press, Oxford

21. Ohsawa M (2011) Shakai wa Taezu Yume wo Miteiru. Asahi Shuppan, Tokyo [Society Dreams Incessantly. Asahi Shuppan, Tokyo](in Japanese)

22. Ohtsuki H, Hauert C, Lieberman E, Nowak MA (2006) A simple rule for the evolution of cooperation on graphs and social networks. Nature, 441(7092):502-505. doi:10.1038/nature04605

23. Phan TQ, Airoldi EM (2015) A natural experiment of social network formation and dynamics. Proceedings of the National Academy of Sciences 112(21):6595-6600. doi:10.1073/pnas.1404770112

24. Solnit R (2010) A paradise built in hell: The extraordinary communities that arise in disaster. Penguin

25. Wolfenstein M (1957) Disaster: A psychological essay. Free Press and Falcon's Wing Press

26. Yamori K (2011) Saigai heno taiou. In: Disaster Prevention Research Institute, Kyoto University (ed) Shizensaigai to Bousai no Jiten, Maruzen Shuppan, Tokyo [Response to disaster. In: Disaster Prevention Research Institute, Kyoto University (ed) The Encyclopedia of Natural Disaster and Disaster Prevention, Maruzen Shuppan, Tokyo](in Japanese) 